\title{
Displasia segmentaria odontomaxilar. Una rara patología de interés odontológico. Aporte de dos nuevos casos
}

\section{Segmental odontomaxillary dysplasia. A rare pathology of dental interest. Two case reports}

\author{
González $\mathrm{G}^{*}$, Keochgerián V**, García L***, Pedreira E*****, Blanco V****
}

\begin{abstract}
RESUMEN
Se aportan dos nuevos casos de displasia segmentaria odontomaxilar. Ésta es una rara patología del desarrollo que se distingue por presentar defectos madurativos en el hueso, encía y dientes de un cuadrante de la maxila. Clínicamente se observan tres signos clínicos característicos: deformación unilateral de la maxila, agrandamiento gingival ipsilateral y agenesia de uno o dos premolares, raramente del primer molar. En ocasiones se asocian alteraciones faciales tales como borde mucocutáneo del labio superior desdibujado, pigmentación unilateral, eritema, hipopigmentación del labio superior e hipertricosis.

Los casos presentes corresponden: uno, al género femenino y otro, al masculino. El diagnóstico de ambos se realizó en la adolescencia y hasta la fecha se encuentran en tratamiento ortodóncico para subsanar las repercusiones estéticas y funcionales generadas por esta patología.

El objetivo de este trabajo es aportar dos nuevos casos a la casuística internacional estableciendo las características clínicas e histopatológicas más relevantes, comparándolos con otros casos publicados.
\end{abstract}

Palabras clave: Displasia hemimaxilar, agrandamiento gingival unilateral, agenesia unilateral de premolares, asimetría facial, HATS.

\section{SUMMARY}

We report two new cases of segmental odontomaxillary dysplasia (SOMD); a rare developmental pathology characterized by segmental maturation defects in bone, gingiva and teeth at one quadrant of the maxilla. It has three typical clinical signs: unilateral maxilla abnormality, ipsilateral gingival enlargement and one or two premolar agenesis, unusual in a first molar. Sometimes it's associated with facial alterations: mucocutaneous indistinct upper lip border, unilateral pigmentation erythema, hypopigmentation of upper lip and hypertrichosis. Cases presented correspond to male and female patients. Both were diagnosed in adolescence; until present day are under orthodontic treatment in order to minimize the aesthetic and functional disorders caused by this pathology.

The purpose of this article is to report two new cases to international registers, establishing the most relevant clinical and histopathological features and comparing them with published studies.

Key words: Hemimaxillary dysplasia, unilateral gingival enlargement, unilateral premolar agenesis, facial asymmetry, HATS.

* Prof. Titular de la Cátedra de Odontopediatría de la Facultad de Odontología, UdelaR.

** Exencargada de la Cátedra de Anatomía Patológica. Facultad de Odontología, UdelaR. Exdirectora de la Unidad de Salud Bucal Instituto Niño y del Adolescente del Uruguay (INAU).

*** Exprofesor de la Cátedra de Ortodoncia de la Facultad de Odontología, UdelaR.

**** Asistente de la Cátedra de Semiología y Clínica Estomatológica. Facultad de Odontología, UdelaR.

***** Prof. Adjunto de la Cátedra de Semiología y Clínica Estomatológica. Facultad de Odontología, UdelaR. 
Fecha de recepción: 17 de diciembre de 2013.

Aceptado para publicación: 18 de febrero de 2014.

González G, Keochgerián V, García L, Pedreira E, Blanco V. Displasia segmentaria odontomaxilar. Una rara patología de interés odontológico. Aporte de dos nuevos casos. Av. Odontoestomatol 2014; 30 (5): 243-250.

\section{INTRODUCCIÓN}

La displasia segmentaria odontomaxilar es una patología del desarrollo no hereditaria, de carácter no progresivo, que involucra estructuras de origen ectodérmico y mesodérmico de un sector de la región odontomaxilofacial.

Constituye una patología poco frecuente. Según Whitt (1) hasta el 2011 existían 45 casos publicados en trabajos de habla inglesa. Hasta el 2013 Azevedo y col. (2) contabilizaron 50 casos.

Clínicamente se caracteriza por presentar deformación asintomática unilateral de la maxila desde el canino hasta la tuberosidad, con desplazamiento de ambas tablas, agrandamiento gingival y anomalías de las piezas dentarias del cuadrante afectado. Generalmente hay agenesia de uno o de ambos premolares, excepcionalmente del primer molar. También se observa retardo eruptivo de las piezas dentarias involucradas. Radiográficamente el hueso afectado muestra alteración en la orientación de las trabéculas y aspecto de vidrio esmerilado como en otras displasias óseas. Del punto de vista regional, se destaca asimetría facial. No se describen alteraciones sistémicas relacionadas.

En el año 1987, Miles (3) comunicó dos casos en pacientes niños con asimetría facial congénita y agrandamiento unilateral de la maxila y de la encía correspondiente. Por las características antedichas sugirió la denominación de Displasia Hemimaxilofacial. Uno de los casos tenía, además, agenesia de ambos premolares e hipoplasia del esmalte en la dentición temporaria del sector afectado; el otro se asociaba a hipertricosis facial ipsilateral.

En el año 1990, Danforth (4) comunicó 8 casos con hallazgos similares pero sin alteraciones cutáneas. Sugirió el término Displasia Segmentaria Odonto- maxilar enfatizando los cambios odontomaxilares que siempre están presentes.

Nuevas comunicaciones aportaron otros hallazgos unilaterales: discontinuidad del borde bermellón del labio, depresión en la zona cutánea de la cara, nevus pigmentado de Becker, área de hipopigmentación en el labio superior, zona de hiperpigmentación de la piel de la cara del lado afectado, esclerosis ósea del hueso maxilar con alteraciones del trabeculado óseo, seno maxilar disminuido de tamaño, retardo eruptivo de las piezas dentarias en ese sector (5-7), manos con eritemas y múltiples líneas en las palmas (8).

En el año 2004, Welsch y Stein (7) señalaron los cambios dermatológicos que se asocian a esta patología y propusieron una denominación de alternativa utilizando la sigla HATS que, en inglés, resume sus principales características: hemimaxillary enlargement, asymmetry of the face, tooth anomalies and skin findings.

Minett y col. (9) observaron que los cambios cutáneos son más frecuentes en la adolescencia que en la niñez, llegando a la conclusión que esta patología del desarrollo no es estática sino que con el paso de los años se expresan nuevas alteraciones.

El objetivo de este trabajo es aportar dos nuevos casos a la casuística internacional estableciendo las características clínicas e histopatológicas más relevantes, comparándolos con otros casos publicados.

\section{Caso 1}

Paciente masculino, raza blanca, de 7 años de edad que consultó de urgencia por tumefacción a nivel del surco nasogeniano izquierdo. Sin antecedentes médicos ni odontológicos destacables. 
Al examen de la zona motivo de consulta se constató pieza dentaria 63 sin caries, con gangrena pulpar, hipoplasia del esmalte y severa anomalía de forma. La zona gingival vestibular se encontraba edematosa y dolorosa a la palpación, drenando exudado serosanguinolento. Además, las piezas 61 y 62 estaban presentes. Los molares temporarios 64 y 65, presentaban corona clínica reducida. El primer molar permanente de ese sector estaba clínicamente ausente. En el cuadrante superior izquierdo, se observó ensanchamiento vestibulopalatino del maxilar, más notorio hacia la tabla vestibular. La deformación ósea se acompañaba de un aumento de espesor de la fibromucosa correspondiente; estas alteraciones determinaron la ocupación de parte del espacio interoclusal (Figs. 1A y B).

Se realizaron estudios radiográficos apicales y una ortopantomografía que mostraron la presencia de un diente supernumerario en la línea media y anomalías diversas en la pieza 63, tales como hipoplasia del esmalte en la corona (esmalte muy delgado), amplia cámara pulpar con único conducto muy amplio y ensanchamiento importante del ligamento periodontal (Fig. 1C).

Se implementó un tratamiento de urgencia con antibioterapia y eliminación de la pieza 63. En segunda instancia se extrajeron los incisivos temporarios en persistencia y el diente supernumerario con la finalidad de eliminar trabas eruptivas.

La pieza 21 se encontraba en una posición cercana a fosas nasales. El germen del segundo premolar estaba ubicado intraóseo en posición alta, mientras que había agenesia del primer premolar. El primer molar permanente tenía retraso en su desarrollo y en su erupción (Fig. 1C). El seno maxilar izquierdo se hallaba disminuido de tamaño comparándolo con el derecho.

Las demás estructuras óseas, dentarias y gingivales de otros sectores de la cavidad bucal se encontraban sin particularidades.

Otros hallazgos clínicos fueron: asimetría facial, desvío de la línea media hacia la derecha, leve estrabismo del ojo izquierdo, nevus pigmentado a la altura del reborde orbitario inferior izquierdo y borde bermellón labial superior desdibujado (Fig. 2A).

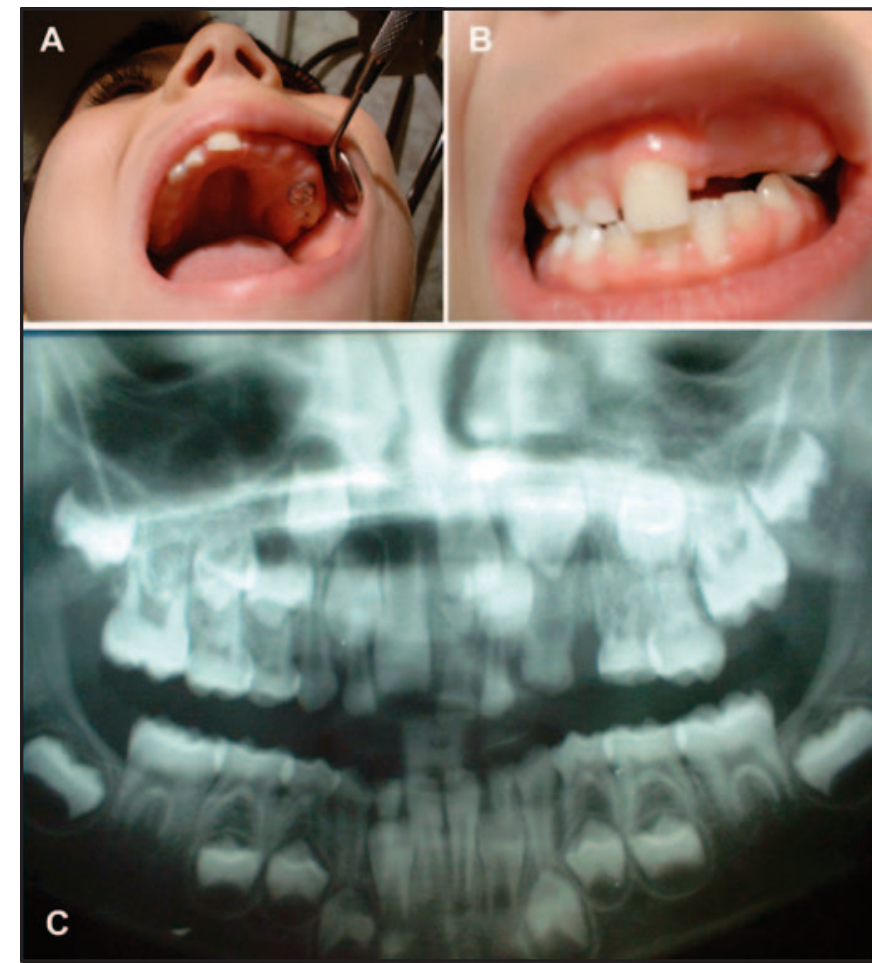

Fig. 1. Caso 1. Aspecto clínico y radiográfico iniciado el tratamiento. A) Ensanchamiento del hemimaxilar superior izquierdo. Presencia de molares 64 y 65. Se han extraído los incisivos temporarios 61 y 62. B) Crecimiento gingival hacia el espacio interoclusal. C) OPT. Anomalía dentaria a nivel del 63. Retenciones del 21 y 22, supernumerario en línea media, agenesia germen del

24, reabsorción externa de la raíz del 64, retardo eruptivo 26.

Durante 6 meses se controló el trayecto eruptivo del 21 hasta que erupcionó y se derivó al ortodoncista.

Hasta ese momento no se contaba con diagnóstico de su patología. Más adelante se realizó interconsulta con patólogos llegándose al diagnóstico de displasia segmentaria odontomaxilar. En ese momento, el paciente contaba con 15 años.

Durante el tratamiento ortodóncico se ubicó al canino superior izquierdo permanente mediante la realización de una descubierta y tracción Sin embargo el hueso alveolar no acompañó ese desplazamiento dentario (Figs. 2B y C).

Se realizó estudio histopatológico del tejido gingival removido al realizar la descubierta del 23. Se informa hiperplasia fibroepitelial de la mucosa gingival, con sectores de profundización del epitelio basal 


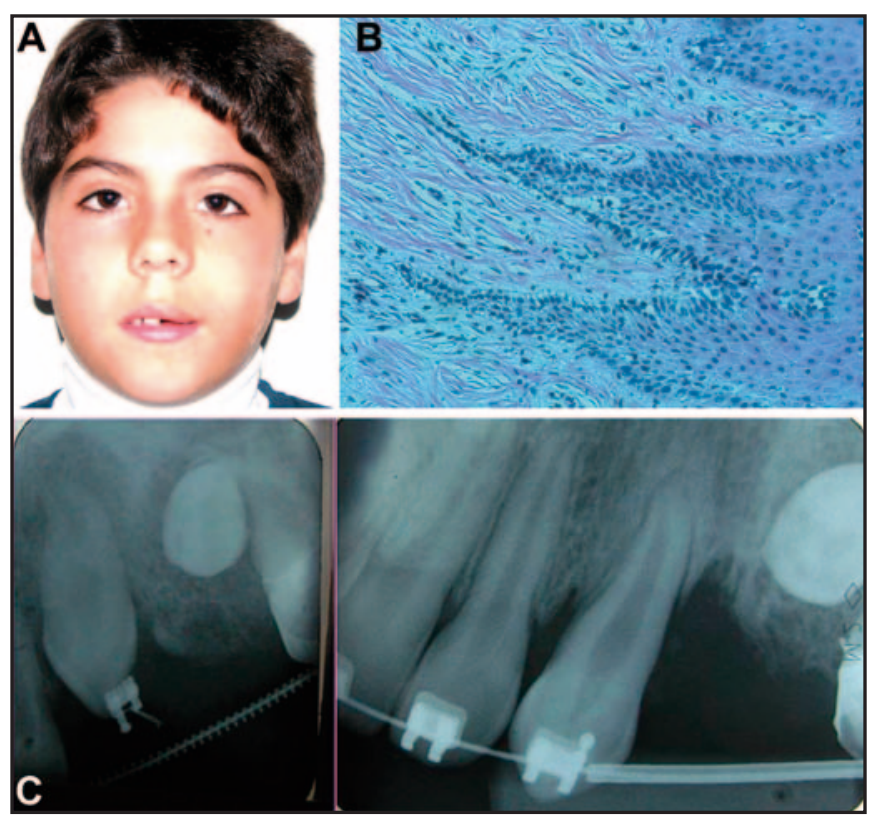

Fig. 2. Caso 1. A) Aspecto clínico. Asimetría facial, nevus pigmentado a nivel infraorbitario, borde bermellón desdibujado. B) Histopatología del tejido gingival. C) Evolución del tratamiento (2/ 2009), pieza 23 ubicada en el reborde maxilar. Obsérvese que el hueso no acompañó la erupción dentaria. Trabeculado óseo de aspecto esclerótico.

hacia el conjuntivo, zonas con polarización invertida de los núcleos de la capa basal y presencia de células claras. También se observan islotes de epitelio odontogénico inactivo en la lámina propia gingival (Fig. 2B).

Se planea completar el tratamiento con la colocación de un implante para rehabilitar el sector premolar.

\section{Caso 2}

Paciente género femenino, raza blanca, 16 años de edad con tratamiento ortodóncico desde los 14 años.

La paciente tenía agenesia de los dos premolares superiores izquierdos. En el mismo cuadrante, se detectó una deformación ósea persistente y asintomática que no se relacionaba con causa conocida. Esto determinó su derivación para que se le realizara una toma biópsica de ese sector; se diagnosticó fibroma osificante.
La deformación ósea se acompañaba de agrandamiento de la fibromucosa del cuadrante involucrado; ésta se presentaba firme y sin signos de inflamación. En el mismo cuadrante había persistencia del molar 65 (Fig. 3A). Las radiografías apicales y ortopantomografía confirmaron la falta de estos gérmenes.

Se indicó tomografía computada, constatándose el ensanchamiento vestíbulo lingual de la maxila en el cuadrante izquierdo, tablas óseas engrosadas, hueso hiperdenso con trabeculado alterado y seno maxilar más pequeño con paredes óseas gruesas (Fig. 3D).

Regionalmente, surgieron otros hallazgos tales como borde mucocutáneo del labio superior desdibujado en el lado afectado y área difusa de pigmentación parda en la piel de la cara cerca del labio superior a nivel unilateral (Fig. 3C).

Del análisis de los aspectos clínicos, imagenológicos e histopatológicos (se revisó la biopsia previa

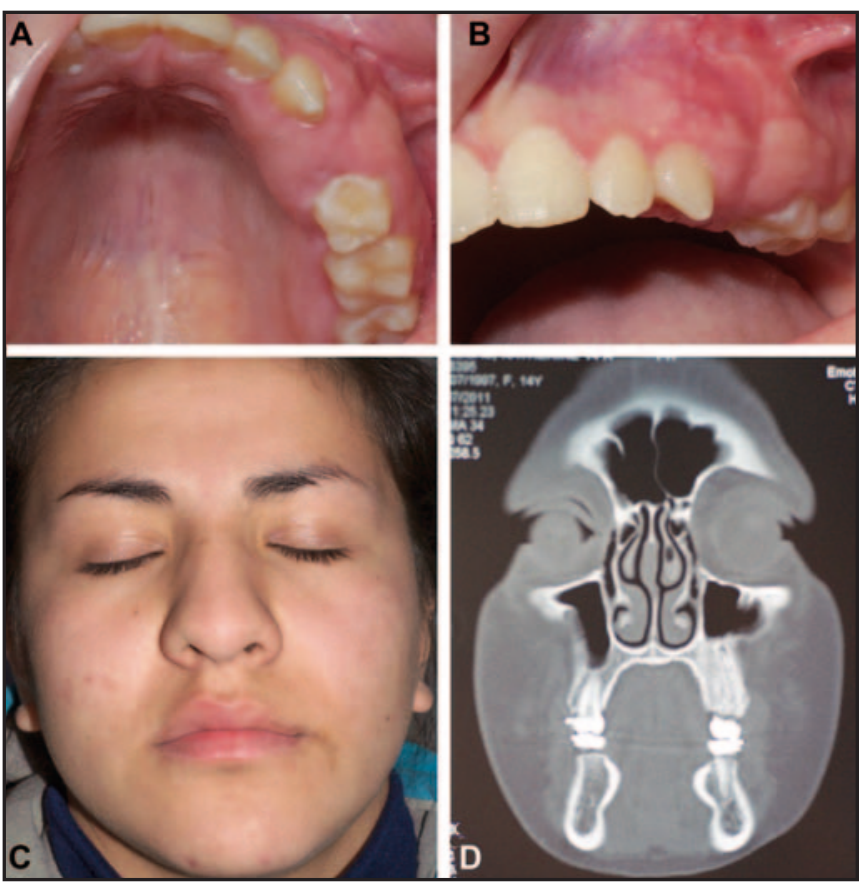

Fig. 3. Caso 2. A y B) Aspecto clínico. Zona motivo de consulta, maxila ensanchamiento unilateral, agrandamiento gingival unilateral, persistencia molar 65. C) Facies. Zona pigmentada, borde bermellón labio superior desdibujado. D) TC corte coronal. Maxila izquierda ensanchada. Tablas óseas vestibular y palatina engrosada. Hueso aspecto esclerótico. Seno maxilar disminuido de tamaño. 
informada como fibroma osificante), surgió el diagnóstico definitivo de displasia segmentaria odontomaxilar.

Con el diagnóstico realizado, se hicieron tomas biópsicas de la fibromucosa y del hueso a tres niveles de profundidad diferentes. La fibromucosa mostró hiperplasia fibroepitelial sin ninguna particularidad. En el hueso, los hallazgos morfológicos fueron similares en las tres tomas, mostrando trabéculas óseas irregulares sin ribete osteoblástico, líneas de aposición reversas y tejido conjuntivo fibroso con sectores vasculares.

\section{DISCUSIÓN}

La displasia segmentaria odontomaxilar es una anomalía del desarrollo poco frecuente. Los casos que aquí se presentan son los primeros comunicados en el Uruguay.

La causa de esta patología es poco clara. Packota (6) sugirió un trastorno del desarrollo en útero de causa local. Otros autores (10) han sugerido causa bacteriana o viral como factores iniciadores. Para Jones (11) podría estar relacionada con una mutación postcigótica, que resulta en un mosaicismo genético y fenotípico del hueso y de la piel.

El diagnóstico es, casi siempre, en la niñez y en la primera década de la vida (12), raramente al nacimiento. Para Whitt y col. (1) se realiza en una edad promedio de 9 años. Los dos casos aquí presentados fueron diagnosticados en la adolescencia, pero los cambios clínicos se habían detectado en la niñez, sin haberse llegado al diagnóstico correcto. En el caso 1, la primera consulta fue a los 7 años de edad y recién a los 15 años se llegó al diagnóstico definitivo debido a la consulta interdisciplinaria. El segundo caso fue catalogado como un fibroma osificante. Para algunos autores es frecuente el error diagnóstico en esta patología. Por este motivo se ha sugerido la posibilidad de una mayor prevalencia que la registrada (12). En muchas ocasiones solo se tienen en cuenta algunos de los hallazgos sin considerar que el correcto diagnóstico se logra analizando todos los cambios presentes, tanto los clínicos locales como los regionales, los imagenológicos y los histopatoló- gicos. Estos últimos no son por sí mismo patognomónicos.

En términos generales, se considera que esta alteración se da por igual en ambos géneros (12). Para algunos $(1,6)$ la frecuencia es mayor en el género masculino. Aquí corresponden uno al género femenino y otro al masculino.

En cuanto a los cuadrantes afectados, la distribución es similar (1). En los dos casos presentes, la ubicación fue en el cuadrante izquierdo.

Del punto de vista clínico, en el caso 1 se observó leve asimetría facial; en el caso 2 esta asimetría no es notoria. Otros hallazgos presentes en ambos casos fueron borde mucocutáneo del labio superior poco delimitado, seno maxilar del lado afectado más pequeño, agrandamiento gingival unilateral y agenesia dentaria a nivel premolar. En el primer caso había agenesia del primer premolar y, en el caso 2, faltaban ambos. El caso 1 tenía anomalías dentarias de número (agenesia de un premolar y un supernumerario en línea media), anomalías de forma en el canino temporario, anomalías estructurales en todas las piezas temporarias del sector y retraso de la erupción de los permanentes correspondientes. También se detectó reabsorción externa radicular en los dos molares temporarios en persistencia Otro hallazgo fue la presencia de un diente supernumerario (mesiodens). Es de destacar que en la bibliografía consultada, no se consigna la presencia de dientes supernumerarios, por lo que se deduce que esta anomalía no es común.

La paciente del caso 2 en el momento de la consulta, tenía persistencia del segundo molar temporario, agenesia de ambos premolares y el resto de las piezas dentarias permanentes erupcionadas. Ninguno de los dientes del cuadrante mostró alteraciones estructurales ni morfológicas.

Otro hallazgo clínico presente en los dos casos y concordante con muchas de las comunicaciones, fue la disminución del tamaño del seno maxilar ipsilateral. Según Whitt y col., esta anomalía está presente en el $49 \%$ de los casos (1).

Los cambios en la piel de la cara están asociados en el 28\% de los pacientes (1). En el caso 1, se presentó 
un nevus pigmentado pequeño que podría estar relacionado con la Displasia segmentaria Odontomaxilar. En el caso 2, se observó una pigmentación melánica difusa, de poca extensión, cerca del labio inferior.

Ambos pacientes tenían el borde bermellón desdibujado.

El estudio histopatológico de los tejidos gingivales mostró cambios morfológicos diferentes en ambos casos. En el caso 2, se observó aspecto microscópico semejante a las hiperplasias gingivales generalizadas de tipo fibroso ocasionadas por otros factores, concordando con lo comunicado en la mayoría de las publicaciones.

En cambio, el caso 1 presentó alteraciones microscópicas particulares tales como: rosetas y cordones de epitelio odontogénico inactivo en la lámina propia gingival, profundizaciones de los brotes interpapilares en forma de cordones delgados, polarización invertida y empalizamiento de los núcleos de las células basales con presencia de células claras epiteliales focalizadas en sectores de la basal. Estos cambios recuerdan a los observados en las lesiones odontogénicas periféricas de tipo hamartomatoso. En dos de los casos comunicados por Whitt (1), se describen formaciones semejantes a quistes de la lámina dental que regresaron con el paso de los años.

Con respecto a la morfología del hueso examinado, es similar a lo descrito en todos los casos. Se trata de un hueso displásico dispuesto en trabéculas grue- sas, sin ribete osteoblástico y líneas de aposición en disposición reversa. Entre estas trabéculas se dispone tejido conjuntivo sin elementos inflamatorios y abundante vascularización. Microscópicamente debe diferenciarse de las lesiones fibroóseas de los maxilares.

La displasia segmentaria odontomaxilar forma parte de un grupo de patologías del área bucomaxilofacial que se presentan con afectación unilateral. Los elementos más relevantes que hacen al diagnóstico diferencial entre esta patología y otras de presentación similar como la odontodisplasia regional, la displasia fibrosa y la hiperplasia hemimaxilar se sintetizan en las tablas 1,2 y 3.

Con respecto al tratamiento, esta entidad constituye un desafío. Las repercusiones funcionales y estéticas solo pueden ser resueltas por el trabajo multidisciplinario. Los casos aquí presentados fueron diagnosticados por patólogos, mientras que el tratamiento fue llevado a cabo por odontopediatras, cirujanos y ortodoncistas.

Queda la interrogante de la respuesta del hueso frente a la colocación de implantes. Se ha comunicado buen resultado frente a la rehabilitación con implantes $(1,10)$. La duda se establece debido a que en el caso 1 el hueso no acompañó la erupción del canino superior izquierdo durante la tracción ortodóncica. Esto nos demuestra que el comportamiento biológico de ese tejido óseo es diferente al del hueso normal. La respuesta reparativa de ese hueso podrá ser evaluada luego de años de controles clínicos e imagenológicos de los implantes colocados.

\begin{tabular}{|l|l|l|c|}
\hline \multicolumn{3}{|c|}{ TABLA 1 } & Historia médica \\
\hline & Diagnóstico & Historia natural & S/p \\
\hline Displasia segmentaria odontomaxilar & Niñez-adolescencia & No progresivo & $\mathrm{S} / \mathrm{P}$ \\
\hline Odontodisplasia regional & Niñez & No progresivo & + \\
\hline Hipertrofia hemifacial congénita & Al nacimiento & $\begin{array}{l}\text { Progresivo hasta } \\
\text { finalizar el desarrollo }\end{array}$ & \begin{tabular}{l} 
S/P \\
\hline Displasia fibrosa monostótica
\end{tabular} \\
\hline
\end{tabular}




\begin{tabular}{|c|c|c|c|c|c|c|c|}
\hline \multicolumn{8}{|c|}{ TABLA 2} \\
\hline & Etiología & Asimetría & Hipertricosis & $\begin{array}{l}\text { Pigmentaciones } \\
\text { faciales }\end{array}$ & $\begin{array}{l}\text { Agrandamiento } \\
\text { gingival }\end{array}$ & $\begin{array}{l}\text { Deformación } \\
\text { ósea }\end{array}$ & Topografía \\
\hline $\begin{array}{l}\text { Displasia } \\
\text { segmentaria } \\
\text { odontomaxilar }\end{array}$ & $\begin{array}{l}\text { Trastorno del } \\
\text { desarrollo } \\
\text { causa } \\
\text { desconocida }\end{array}$ & $\begin{array}{l}\text { Unilateral } \\
\text { maxilar } \\
\text { y facial }\end{array}$ & $\begin{array}{l}\text { Presente en } \\
\text { algunos } \\
\text { casos }\end{array}$ & Unilateral facial & + & + & $\begin{array}{l}\text { Maxilofacial } \\
\text { unilateral }\end{array}$ \\
\hline $\begin{array}{l}\text { Odontodisplasia } \\
\text { regional }\end{array}$ & $\begin{array}{l}\text { Trastorno del } \\
\text { desarrollo } \\
\text { causa } \\
\text { desconocida }\end{array}$ & - & - & - & Ausente & - & $\begin{array}{l}\text { Piezas } \\
\text { dentarias, } \\
\text { más común } \\
\text { un cuadrante }\end{array}$ \\
\hline $\begin{array}{l}\text { Hipertrofia } \\
\text { hemifacial } \\
\text { congénita }\end{array}$ & $\begin{array}{l}\text { Anomalías } \\
\text { cromosómicas }\end{array}$ & $\begin{array}{l}\text { Unilateral } \\
\text { facial } y / o \\
\text { corporal }\end{array}$ & + & - & + & + & $\begin{array}{l}\text { Hemicuerpo } \\
\text { varios órganos } \\
\text { afectados }\end{array}$ \\
\hline $\begin{array}{l}\text { Displasia } \\
\text { fibrosa } \\
\text { monostótica }\end{array}$ & $\begin{array}{l}\text { Trastorno del } \\
\text { desarrollo } \\
\text { causa } \\
\text { desconocida }\end{array}$ & $\begin{array}{l}\text { Unilateral } \\
\text { ambos } \\
\text { maxilares }\end{array}$ & - & - & Ausente & + & $\begin{array}{l}\text { Maxilares, } \\
\text { más en } \\
\text { maxila }\end{array}$ \\
\hline
\end{tabular}

TABLA 3

\begin{tabular}{|l|l|l|l|}
\hline Alteraciones dentarias & Estructurales & No estructurales & Eruptivas \\
\hline $\begin{array}{l}\text { Displasia segmentaria } \\
\text { odontomaxilar }\end{array}$ & $\begin{array}{l}\text { Dentición temporaria: } \\
\text { hipoplasia del esmalte. } \\
\text { Alteraciones dentinarias, } \\
\text { reabsorción dentinaria, } \\
\text { cálculos pulpares. }\end{array}$ & $\begin{array}{l}\text { Coronas y raíces más largas } \\
\text { en ambas denticiones, } \\
\text { agenesia dentaria: en } \\
\text { premolares, raro otras piezas }\end{array}$ & Retardo eruptivo \\
\hline $\begin{array}{l}\text { Odontodisplasia } \\
\text { regional }\end{array}$ & $\begin{array}{l}\text { Todos los tejidos dentales, } \\
\text { afectados, capuchón con } \\
\text { alteraciones y cálculos pulpares. }\end{array}$ & - & Retardo eruptivo \\
\hline $\begin{array}{l}\text { Hipertrofia hemifacial } \\
\text { congénita }\end{array}$ & - & $\begin{array}{l}\text { Agrandamiento mesiodistal } \\
\text { de las coronas temporarias. } \\
\text { Raíces más largas }\end{array}$ & $\begin{array}{l}\text { Erupción precoz } \\
\text { de los } \\
\text { permanentes. }\end{array}$ \\
\hline $\begin{array}{l}\text { Displasia fibrosa } \\
\text { monostótica }\end{array}$ & - & - & - \\
\hline
\end{tabular}

En conclusión, la displasia segmentaria odontomaxilar es una alteración de interés para el odontólogo debido a las repercusiones estéticas y funcionales que provoca. El diagnóstico definitivo surge del análisis de los hallazgos clínicos, imagenológicos e histo- patológicos. La resolución de estos casos constituye un desafío para la profesión odontológica siendo fundamental el trabajo multidisciplinario. Cada caso debe ser tratado en forma particular considerando los cambios que se presentan. 
La comunicación de nuevos casos ayudará al mejor conocimiento de esta rara patología evitando errores diagnósticos que pueden llevar a tratamientos quirúrgicos innecesarios y muchas veces mutilantes para el paciente.

\section{BIBLIOGRAFÍA}

1. Whitt JC, Rokos JW, Dunlop CH L, Baker BF. Segmental odontomaxillary dysplasia: report of a series of 5 cases with long-term follow-up. Oral Surg Oral Med Oral Pathol Oral Radiol Endod 2011;112:e29-e47

2. S Azevedo R, J da Silveira L, Flávio M Moliterno L, Maria M A Miranda A, P de Almeida O, R Pires F. Segmental odontomaxillary dysplasia: report of a case emphasizing histopathological, immunohistochemical and scanning electron microscopic features. J Oral Sci 2013;55(3):259-62.

3. Miles DA, Lovas JL, Cohen MM Jr. Hemimaxillofacial dysplasia: a newly recognized disorder of facial asymmetry, hypertrichosis of the facial skin, unilateral enlargement of the maxilla, and hypoplastic teeth in two patients. Oral Surg Oral Med Oral Pathol 1987;64:445-8.

4. Danforth RA, Melrose RJ, Abrams AM, Handlers JP. Segmental odontomaxillary dysplasia. Report of eight cases and comparison with hemimaxillofacial dysplasia. Oral Surg Oral Med Oral Pathol 1990;70:81-5.

5. De Salvo MS, Copete MA, Riesenberger RE, Cleveland DB, Chen SY. Segmental odontomaxillary dysplasia (hemimaxillofacial dysplasia): case report. Pediatr Dent 1996;18:154-6.

6. Packota GV, Pharoah MJ, Petrikowski CG. Radiographic features of segmental odontomaxillary dysplasia: a study of 12 cases. Oral Surg
Oral Med Oral Pathol Oral Radiol Endod 1996; 82:577-84.

7. Welsch MJ, Stein SL. A syndrome of hemimaxillary enlargement, asymmetry of the face, tooth abnormalities, and skin findings (HATS). Pediatr Dermatol 2004;21:448-51.

8. Koening Lk, Lynch DP, Yancey K B. Segmental odontomaxillary presenting with facial hipertricosos, comisural lip clefting, and hyperlinear palms. Pediatr Dermatol 2008;25:491-2.

9. Minett CP, Daley TD. Hemimaxillofacial dysplasia (segmental odontomaxillary dysplasia): case study with 11 years of follow-up from primary to adult dentition. J Oral Maxillofac Surg 2012;70: 1183-91. Epub 2011 Jul 20.

10. Becktor KB, Reibel J, Vedel B, Kjaer I. Segmental odontomaxillary dysplasia: clinical, radiological and histological aspects of four cases. Oral Dis 2002;8:106-10.

11. Jones AC, Ford MJ. Simultaneous occurrencia of segmental odontomaxillary dysplasia and Becker's nevus. J Oral Maxillofac Surg 1999;57: 1251-4.

12. González-Arriagada WA, Vargas PA, FuentesCortés R, Nasi-Toso MA, Lopes MA. Segmental odontomaxillary dysplasia: report of 3 cases and literature review. Head Neck Pathol 2012;6(2): 171-7.

\section{CORRESPONDENCIA}

Verónica Keochgerián

Camino Castro, 129 bis

CP 11900 Montevideo. Uruguay

Correo electrónico: verokeoc@hotmail.com. 